\title{
Profesionales, amateurs y gremialistas. Trayectorias y entradas a la política de los "agrodiputados" en el Congreso argentino'
}

Gabriel Levita

\section{Resumen}

Este artículo indaga desde una sociología política de las elites acerca de las entradas a la política partidaria en la Argentina contemporánea a través del estudio de las trayectorias sociales, gremiales y políticas de los diputados nacionales electos en 2009 provenientes de las dirigencias de organizaciones agropecuarias, conocidos como "agrodiputados". Lo hace desde una perspectiva cualitativa y utilizando fuentes primarias - entrevistas en profundidad - y secundarias.

Mostramos cómo estos diputados, que han sido considerados a partir de sus propiedades comunes, constituyen en realidad un agregado heterogéneo que agrupa a tres perfiles de actores. Analizamos sus ingresos a la política partidaria y la reconversión de recursos en capital político. Mostramos cómo el provenir "desde afuera" sólo valió como recurso al comienzo de su carrera y cómo luego terminaron imponiéndose las lógicas de la política profesional.

Palabras clave: Diputados. Entradas a la política. Capital político. Carreras políticas. Argentina.

\section{Resumo}

Este artigo explora a partir de uma sociologia política das elites sobre as entradas na política partidária na Argentina contemporânea através do estudo das trajetórias sociais, sindicais e políticas dos deputados nacionais eleitos em 2009 provenientes das dirigencias das organizações agrícolas, conhecidos como "agrodeputados". Ele faz isso de uma perspectiva qualitativa e usando fontes primárias - entrevistas em profundidade - e fontes secundárias. Mostramos como esses deputados, que foram considerados a partir de suas propriedades comuns, constituem na reali-

I Una versión preliminar de este trabajo fue presentada en el $11^{\circ}$ Encontro da Associação Brasileira de Ciência Política, Curitiba, 2018. Agradezco las sugerencias y comentarios de Wellington Nunes, Mariana Gené, Martín Armelino, Luis M. Donatello y de los evaluadores anónimos de la Revista Política E Sociedade.

2 Doctor en Ciencias Sociales y en Estudios Políticos por la Universidad de Buenos Aires y la École des Hautes Études en Sciences Sociales. Investigador asistente del CONICET en el Instituto de Problemas Nacionales y profesor adjunto en el Departamento de Planificación y Políticas Públicas de la Universidad Nacional de Lanús (UNLa). E-mail: glevita@unla.edu.ar. Commons. Com essa licença você pode compartilhar, adaptar, para qualquer fim, desde que atribua a autoria da obra, forneça um link para a licença, e indicar se foram feitas alterações. 
dade um agregado heterogêneo que agrupa três perfis de atores. Analisamos suos ingresos a la política partidária e a reconversão de recursos em capital político. Mostramos como a vinda "de fora” só se aplicou como um recurso no início de sua carreira e depois acabou impondo a lógica da política profissional.

Palavras-chave: Deputados. Entradas na política. Capital político. Carreiras políticas. Argentina.

\section{Introducción}

El ingreso a la política partidaria por parte de quienes acumularon prestigio y reconocimiento en otras actividades profesionales por fuera de los partidos políticos, ha sido visto tanto desde una mirada positiva en términos de "renovación" como, al mismo tiempo, criticado por contribuir a un supuesto deterioro de la calidad de las instituciones. En la Argentina en particular, a pesar de tratarse de un fenómeno de larga data, el tema ha cobrado resonancia en los últimos años con los efectos de la crisis política y social de 2001-2002 como telón de fondo. Ésta acentuó el deterioro de la matriz bipartidista entre el Partido Justicialista y la Unión Cívica Radical y profundizó la fragmentación de los partidos - especialmente los del espacio no peronista - con la consiguiente aparición de nuevas fuerzas más pequeñas (TORRE, 2003; MUSTAPIC, 2013). En ese contexto, frente al creciente descrédito de la política tradicional y del rol del parlamento, los costos de ingreso al campo político disminuían y los candidatos provenientes "desde afuera" se convertían en instrumentos de legitimación (VOMMARO, 2017).

Al mismo tiempo, la entrada al Congreso de representantes corporativos posee una extensa tradición en la Argentina, aunque los trabajos centrados en las últimas décadas han abordado principalmente el caso de los sindicalistas (DAMIN, 2011; ARMELINO, 2016) y de los empresarios (DONATELLO, 2017). Durante el período 2003-2011, un $14,5 \%$ de los diputados nacionales provenía del mundo empresarial -empresarios, gerentes o representantes patronales- y un 3,4\% del sindicalismo (DONATELLO; LEVITA, 2017) ${ }^{3}$.

3 Para el conjunto de los países del Mercosur (Argentina, Brasil, Paraguay y Uruguay) los promedios son de 20,8\% para el mundo empresarial y 10,3\% para el sindical (DONATELLO; LEVITA, 2017). 
En este artículo indagamos acerca de la llegada a la Cámara de Diputados tras las elecciones legislativas de 2009 de un conjunto de actores definidos en su momento como los "agrodiputados": representantes de las principales organizaciones gremiales ruralistas que se habían candidateado por los partidos de oposición luego de un duro enfrentamiento entre el gobierno nacional y el sector agropecuario que había tenido lugar el año anterior ${ }^{4}$.

Los agrodiputados que asumieron sus bancas en el Congreso han sido considerados a menudo, tanto por la prensa como por los escasos estudios académicos existentes sobre el tema, como un conjunto relativamente homogéneo, definido a partir de su llegada al parlamento gracias al o como consecuencia del conflicto con el campo. Mientras que para Grass (2012) representan la efectividad del empresariado rural para intervenir en la política, aunque la incorporación de representantes haya sido inorgánica y atomizada, para Del Cogliano (2016) fueron los partidos quienes convocaron a los dirigentes del agro para mejorar su performance electoral. En este sentido, la vinculación entre los miembros de las organizaciones rurales y la política partidaria ha sido señalada como débil y problemática (LATTUADA, 2006; HORA, 2010).

En este artículo nos preguntamos ¿Cuáles fueron las condiciones de ingreso a la competencia partidaria de los agrodiputados? ¿Qué recorridos realizaron a partir de su llegada al Congreso? Y desde una mirada más amplia centrada en la sociología de política de las elites, ¿qué nos dice este fenómeno acerca de las características que presentan los ingresos a la política en la Argentina contemporánea?

El presente artículo indaga desde una perspectiva cualitativa las trayectorias sociales, gremiales y políticas de los diputados nacionales argentinos electos en 2009 que provenían de las dirigencias de las

4 Entre marzo y julio de 2008 se dio en la Argentina un conflicto entre el gobierno nacional presidido por Cristina Fernández de Kirchner y un amplio sector de productores agropecuarios representados en distintas organizaciones ruralistas. Comenzó siendo una cuestión fiscal y sectorial, pero se politizó rápidamente, quedando atravesado por las lógicas partidarias preexistentes (LEIRAS; CRUZALEGUI, 2009; NARDACCHIONE; TARABORELLI, 20I0). 
organizaciones ruralistas. Se trata de once individuos ${ }^{5}$ cuyos ingresos a la política analizamos a partir de diferentes dimensiones, valiéndonos de fuentes primarias - entrevistas en profundidad - y secundarias, como sus páginas web personales, currículums en línea, bases de datos y artículos y entrevistas de prensa, entre otras. El trabajo realiza un análisis diacrónico a partir de sus trayectorias, centrándose en sus recorridos entre los años 2009 y 2019.

Mostramos cómo un conjunto de actores considerados tanto por la prensa como por trabajos académicos anteriores a partir de sus características homogéneas y sus propiedades comunes constituye en realidad un agregado heterogéneo que agrupa a tres perfiles de actores, con diferentes trayectorias sociales, gremiales y políticas, distintos modos de ingreso a la política partidaria y disímiles formas de habitar el Congreso durante sus mandatos.

El artículo consta de tres secciones y unas conclusiones. En la primera parte desarrollamos el enfoque de la sociología política para el análisis del fenómeno. El segundo apartado se centra en el estudio de las trayectorias sociales y carreras políticas de los once agrodiputados, mientras que la tercera sección realiza una comparación entre los distintos recorridos. Finalmente, las reflexiones finales recapitulan los principales hallazgos y proponen una hipótesis interpretativa desde el punto de vista sociológico.

\section{Los ingresos a la política partidaria como problema desde la sociología política de las elites}

En la Argentina, la sociología política de las elites se interesó en los últimos años por los recorridos a través de los cuales los políticos profesionales y los altos funcionarios del Estado llegaron a sus posiciones tanto a nivel nacional como provincial y municipal. Así, el reclutamiento

5 La contabilidad difiere en los diversos trabajos sobre el tema. Para Grass (2012) son dieciocho, Del Cogliano (2016) contabiliza doce y De Luca y Malamud (2010) señalan once, al igual que Tula y De Luca (20I I). Desde la prensa, la mayoría de los artículos coincide en sumar once. Véanse "La cosecha de los agrodiputados" en 30/06/2009 (DANDAN, 2009), "Los agrodiputados van divididos al Congreso" en 30/I I/2009 (AGROSÍTIO, 2009); "Los agrodiputados pelean espacios clave" en 08/I2/2009 (LA NACIÓN, 2009) y "Sólo quedarán 2 “agrodiputados' de los II que entraron en 2009” en 29/I0/2013 (CLARÍN, 20 I3). 
político ha sido estudiado para el nivel legislativo nacional (CANELO, 2011; RODRIGO, 2014; LEVITA, 2017) y subnacional (ORTIZ DE ROZAS, 2011; LANDAU, 2015; MELLADO, 2016), tanto como para los gabinetes nacionales (HEREDIA GENÉ; PERELMITER, 2012; PERELMITER, 2012; GENÉ, 2019; CANELO, 2019) y los poderes ejecutivos provinciales (VOMMARO; ARMESTO, 2015; SOSA, 2019; LASCURAIN, 2019).

En este contexto, la pregunta por los ingresos "desde afuera", es decir de quienes han acumulado recursos y reconocimientos en otras actividades profesionales distintas a la política partidaria, ha estado más enfocada en el poder ejecutivo y en las trayectorias de ministros, secretarios y subsecretarios que hacen valer recursos extrapartidarios en su acceso a la alta función pública (GIORGI, 2014). El ámbito parlamentario ha recibido menos atención, destacándose la entrada de actores desde el mundo empresarial y sindical (DONATELLO; LEVITA, 2017).

Desde el punto de vista teórico, las conceptualizaciones han girado en torno a las entradas al campo político y a los capitales con que todo actor ingresa y se profesionaliza (Bourdieu, 1981), si bien entre profesionales y profanos se da un continuo con diversos tipos de modalidades de dedicación a la política (OFFERLÉ, 1996, 2011). A su vez, distinguen entre los ingresos apoyados en capitales mayormente individuales -recursos ligados a la propia persona- y aquellos sustentados en capitales colectivos, es decir, en recursos organizacionales de los partidos (GAXIE, 2004, p. 75-85; OFFERLÉ, 2004, p. 43-47).

Las entradas pueden realizarse a partir de estrategias de reconversión en las que distintos tipos de recursos se transforman en capital político (TISSOT, 2004), aunque otras especies de capital -la notoriedad, el carisma y la familia, por ejemplo- también pueden hacerse valer en la competencia partidaria (JOIGNANT, 2012). Asimismo, Boltanski (1973) sostiene a partir de la idea de multiposicionalidad que los actores dominantes en un campo también tenderán a ocupar las posiciones más privilegiadas en los otros en que participe. Esta interdependencia entre la política y otros espacios supone que los factores extrapolíticos son centrales en la explicación de las carreras políticas (GAXIE, 2002). 
Este tipo de ingresos supone una movilización de recursos materiales y simbólicos por fuera de los partidos. Dichos capitales extrapoliticos se activan en la arena política reconvirtiéndose, eventualmente, en capitales políticos. También aquí es posible distinguir estructuras de capitales extrapolíticos más individuales o más colectivas (LEVITA, 2019). Un primer tipo de capital extrapolítico proviene entonces del reconocimiento público de un cierto prestigio basado en la figura personal y originado en la actividad profesional de la que participan. Entendido por los actores en términos de credibilidad, honestidad, experiencia, habilidad, virtud, etc., puede originarse en distintos ámbitos como el periodismo, el deporte, el arte o el espectáculo y tiene una de sus condiciones de posibilidad en el hecho de que son portados por personajes con presencia en los medios de comunicación.

Un segundo tipo podemos definirlo como colectivo, en tanto el reconocimiento se origina en los recursos de una organización a la que se pertenece o perteneció y que, en algunos casos, es la que se pone en valor a través del actor en cuestión. La posición se la debe al grupo o institución de la que provienen, como puede ser el caso de los sindicalistas, los miembros de organizaciones patronales, los integrantes de ONG y los representantes de movimientos sociales, entre otros, más allá de que al momento de ser reclutados se encuentren o no vinculados a dicha organización.

Estos recursos se combinan de múltiples formas y permiten vincular los distintos tipos de ingreso al parlamento con diferentes maneras de habitarlo durante sus mandatos y con las carreras políticas a que dan lugar. En el caso de los agrodiputados, dan cuenta de diferentes perfiles que explican sus distintos desempeńos en la Cámara y sus recorridos posteriores.

\section{Del campo al Congreso. Las trayectorias de los agrodiputados}

En el contexto del conflicto entre el gobierno y el sector agropecuario, la participación en organizaciones ruralistas constituyó el principal capital movilizado por los agrocandidatos en su llegada a la Cámara de Diputados en $2009^{6}$. Se trata de un tipo de reconocimiento originado en los recursos

6 La Honorable Cámara de Diputados de la Nación tiene 257 miembros elegidos por períodos de cuatro años en representación de cada una de las 23 provincias argentinas y de la Ciudad Autónoma de Buenos Aires, que 
de la entidad a la que pertenecían o pertenecieron y que es el que se puso en valor al candidatearse el actor en cuestión. Su posición se la debían al grupo o institución de la que provenían, más allá de que al momento de ser reclutados se encontraran o no vinculados a ella. Así, pertenecer a la Federación Agraria Argentina (FAA), a la Sociedad Rural Argentina (SRA) o a Confederaciones Rurales Argentinas (CRA), por ejemplo, constituyó para estos actores un recurso que, en los meses posteriores al conflicto entre el gobierno y el agro, fue susceptible de hacerse valer en su intento de ingresar al Congreso a través de partidos de oposición.

Asimismo, todos poseían un saber hacer representativo, en tanto eran expertos en actividades de representación gremial y poseían, en ese sentido, experiencia en la organización y participación en elecciones, en la negociación por el reparto de recursos materiales y simbólicos o en la mediación entre intereses divergentes, sólo por nombrar algunas de las destrezas y saberes prácticos con los que, quien alcanza altos cargos en un partido, organización gremial o sindicato, por caso, debe contar en mayor o menor medida.

Junto a los recursos construidos de manera colectiva, también poseían un capital de tipo individual vinculado al reconocimiento mediático cosechado en tanto participantes del conflicto con el gobierno. A pesar de haberlo hecho en muchos casos a título de las organizaciones a las que pertenecían, la dinámica de la mediatización y la participación en entrevistas de prensa y programas televisados hizo que se tratara de un recurso personal, puesto que el conocimiento fue antes de la figura que del conjunto de asociados, individualmente anónimos.

Desde el punto de vista de los selectores, es decir, de quienes seleccionan desde los partidos políticos a los que van a integrar la lista de candidatos, estos postulantes cumplían también con una cuota de

funcionan como 24 distritos electorales. La mitad de las bancas se renuevan cada dos años por medio de un sistema de representación proporcional en el cual se votan en cada distrito listas cerradas de candidatos de un partido político. La distribución de escaños se rige por el método D’Hondt. En los comicios del año 2009 se votaron cargos legislativos nacionales, provinciales y municipales en todo el país y correspondieron al medio término del primer mandato presidencial de Cristina Fernández de Kirchner (2007-20II). En Diputados se renovó la mitad de los representantes de cada provincia. 
“representación simbólica” (BRUĖE; GAXIE, 2014), en tanto ponían en valor la oferta de candidatos del espacio a partir de la inclusión de competidores con atributos positivamente valorados en ese momento determinado por el electorado al que ese partido buscaba captar. Eran vistos como representantes del sector rural al mismo tiempo a partir de su efectiva implantación en la producción agropecuaria y en las organizaciones ruralistas y de su imagen mediática que combinaba provenir del agro con ser considerados empresarios exitosos (HORA, 2010).

Ahora bien, tras los rasgos comunes que compartían estos actores, podemos identificar tres perfiles con diferentes trayectorias sociales, gremiales y políticas, distintos modos de ingreso a la política partidaria y disímiles formas de habitar el Congreso durante sus mandatos. Cada uno de estos tipos supuso una movilización específica de capitales y recursos políticos y extrapolíticos, un vínculo determinado con las elites ruralistas y partidarias y un desempeño político particular.

\section{De vuelta al ruedo: los políticos profesionales}

De acuerdo al planteo clásico de Weber (2007), el ejercicio de la política como profesión es una actividad de tiempo completo, en tanto se vive de ella y para ella. Es decir que garantiza el sustento material al político a condición de que este se aboque a ejercer su profesión política, respectivamente. Sin embargo, existen distintos grados y modalidades de profesionalización (OFFERLÉ, 1996; FERRARI, 2011; GENÉ, 2011; LEVITA, 2015), en las que coexisten diferentes tipos de dedicación a la política, compatibles a su vez con la realización de otras actividades económicas, dada la posibilidad que brinda la temporalidad de la política -signada por la periodicidad de mandatos y elecciones- de vincularse a ella de manera temporaria sin perder por ello la cualidad de político profesional (OFFERLÉ, 1999).

En este primer grupo encontramos a Atilio Benedetti (1955), oriundo de Larroque, Entre Ríos, quien comenzó su militancia política en la Unión Cívica Radical (UCR) al ingresar a la carrera de bromatología en la Universidad Nacional de Entre Ríos. En esa época fue presidente de la Juventud Radical y docente y consejero en la universidad. Con la vuelta de 
la democracia en 1983 resultó electo concejal de Larroque y en 1995 ganó la intendencia. Al mismo tiempo, fue ocupando diversos cargos partidarios en el radicalismo provincial y nacional.

Paralelamente, llegó a ser uno de los mayores productores agropecuarios de la zona y propietario de una empresa de servicios agrícolo-ganaderos para productores más pequeños. Participó en diversas organizaciones empresariales del área, así como en la Bolsa de Cereales de Entre Ríos, la de Buenos Aires y la Bolsa de Comercio provincial, de la que fue su presidente.

En 2009 era presidente del Consejo Provincial de la UCR. Encabezó la boleta de candidatos a diputados nacionales de Entre Ríos por el Acuerdo Cívico y Social (ACyS), una alianza electoral conformada para las elecciones de ese año por la Unión Cívica Radical, la Coalición Cívica - ARI (CC-ARI) ${ }^{7}$ y el Partido Socialista. La lista resultó electa en primer lugar, lo cual le permitió ingresar al Congreso junto a Hilma Ré y Jorge Chemes. Al finalizar su mandato en 2013 fue candidato a senador nacional por la lista de $\mathrm{UNEN}^{8}$, pero quedó en tercer lugar. En 2015 intentó sin éxito ser precandidato a gobernador y luego, con el cambio en el ejecutivo nacional, fue designado director del Banco de la Nación Argentina. Dos ańos más tarde volvió a competir para diputado encabezando la lista de Cambiemos ${ }^{9}$, que triunfó e hizo ingresar a tres candidatos con mandato hasta 2021. En 2019 fue candidato a gobernador, pero quedó segundo.

Por su parte, Gumersindo Alonso (1949) proviene de una familia tradicional de la ciudad de Río Cuarto, Córdoba, dedicada a las actividades agropecuarias y a la cría de caballos de carrera. De allí que, además de abogado y escribano y fuera también presidente del Jockey Club de Río Cuarto. Como productor agropecuario presidió la Sociedad Rural de Río

7 La Coalición Cívica para la Afirmación de una República Igualitaria surgió en 2007 como una unión de partidos en torno al ARI, encabezado por la dirigente Elisa Carrió, proveniente del radicalismo. En 2009 formó parte del ACyS y en 2013 integró el frente UNEN. Desde 2015 forma parte de la alianza oficialista Cambiemos.

8 Coalición electoral surgida en 2013 a partir de múltiples partidos y estructurada en torno a la UCR y a la CC-ARI.

9 Alianza política conformada en 2015 por el PRO, la Unión Cívica Radical (UCR) y la CC-ARI. Llevó como candidato presidencial al líder y fundador del PRO, Mauricio Macri, quien derrotó al postulante kirchnerista en las elecciones de ese año. 
Cuarto y fue delegado por la Confederación de Asociaciones Rurales de la Tercera Zona al Consejo Nacional de CRA.

Ocupó diversos cargos en el PJ cordobés. Fue diputado provincial en la década del 90 y jefe de bloque del oficialismo del entonces gobernador De la Sota hasta que en 1998 lo designaron secretario de Agricultura, Ganadería y Pesca del gobierno nacional de Carlos Menem. Luego fue nombrado secretario de agricultura de la provincia y en 2003 fue elegido diputado nacional por el Partido Nuevo, liderado por el entonces intendente de la ciudad de Córdoba Luis Juez. Al finalizar su mandato en 2007, continuó participando del juecismo a la par de su actividad agropecuaria y su vinculación con CRA. Para las elecciones legislativas de 2009, Alonso encabezó la lista para diputados, ganando su banca hasta 2013 en el marco del Frente Cívico y Social (FCyS) de Juez. Al terminar su mandato no se candidateó para ningún cargo y volvió a dedicarse a las actividades en el campo y a la representación gremial, a la par que siguió vinculado al armado político del FCyS.

Un perfil similar al de estos dos es el de la correntina Josefina Angélica Meabe Ferré de Mathó (1939), quien pertenece a una familia de propietarios agropecuarios y miembros del histórico Partido Liberal de Corrientes. Fue concejal e intendenta de la ciudad de Corrientes y diputada provincial por dos períodos a partir de 2001. Al finalizar su segundo mandato, en 2009, en el contexto del surgimiento de los agrocandidatos, compitió y ganó la senaduría por su provincia en alianza con el radicalismo. Otros trabajos académicos y artículos de prensa la consideran "agrocandidata”, aunque en su caso al Senado.

Para estos auténticos políticos profesionales el conflicto con el campo y el clima electoral generado en torno a los comicios legislativos de 2009 significó una posibilidad de volver a postularse en posiciones expectantes para ingresar al Congreso tras períodos sin ocupar cargos públicos, aunque sí partidarios. Benedetti había dejado la intendencia hacía diez años, aunque continuaba compitiendo dentro del radicalismo entrerriano a la par de su actividad como empresario agropecuario. Alonso había concluido su anterior mandato como diputado nacional y permanecía dentro del espacio político de Juez. Si sumáramos aquí a Meabe, nos encontraríamos 
con una candidata presta a renovar su banca de diputada o a conquistar otro cargo.

En este regreso a los primeros planos de la competencia electoral movilizaron principalmente recursos políticos colectivos y extrapolíticos individuales. Respectivamente, en tanto "hombres de partido", poseían un capital político construido de manera colectiva en torno a la organización partidaria que sustentaba a cada uno, pero además, poseían un reconocimiento mediático de sus figuras personales alcanzado a partir de la cobertura periodística del conflicto de 2008, que significó también un capital a hacer valer en la competencia partidaria. Cada uno encontró una oportunidad de continuar o retornar a la competencia electoral movilizando todos los recursos de un hombre o una mujer de partido junto con el capital novedoso que implicaba el formar parte del mundo rural en la coyuntura del conflicto agrario.

\section{Entrar "por arriba": los amateurs con proyecto}

En otro conjunto de casos el ingreso a la política partidaria y a la competencia electoral se dio recién a partir del conflicto con el campo, independientemente de que tuvieran o no algún contacto con partidos políticos con anterioridad. Canon (1990) propone la categoría de amateur con ambición para designar a estos actores que acceden al Congreso como primer puesto de su carrera política y buscan luego hacerse reelegir o conquistar nuevos cargos, comportándose de modo similar a los políticos profesionales.

Por caso, Juan Casañas (1963) se recibió de ingeniero agrónomo en la Universidad Nacional de Tucumán. Como productor agropecuario de su provincia, se unió a la Asociación Argentina de Consorcios Regionales de Experimentación Agrícola (AACREA) en 2003. En 2008, al calor del conflicto entre el gobierno kirchnerista y el campo, adquirió protagonismo mediático en las protestas y cortes de ruta llevados a cabo en Tucumán y se afilió a la FAA.

Para las elecciones de 2009 fue convocado por dirigentes radicales y peronistas del ACyS a presentarse como primer candidato a diputado nacional, obteniendo su banca por cuatro años. Promediando su mandato 
en 2011, se afilió a la UCR y se presentó como candidato a vicegobernador de Tucumán acompañando al radical José Cano, aunque la fórmula quedó en segundo lugar. Cuando finalizó su período en 2013 volvió a presentarse como candidato para diputado, pero la lista sólo hizo ingresar a los primeros dos y Casańas ocupaba esta vez el tercer puesto. Sin embargo, terminó asumiendo de todas formas, ya que la diputada electa en segundo lugar renunció para asumir como senadora. Durante este segundo mandato se retiró del bloque de Cambiemos en la Cámara Baja y formó junto a una comprovinciana un bloque aparte, pero antes de finalizar su período en 2017 fue designado por el propio gobierno nacional funcionario en el Ministerio de Agroindustria a cargo de Ricardo Buryaile como director del programa Cambio Rural. De esa manera, el gobierno recuperaba un diputado, ya que el suplente de Casańas se incorporó al bloque oficialista. Con la renuncia del ministro a fines de ese año, Casañas también salió de su cargo. En 2019 estuvo cerca de ser candidato a intendente en su provincia.

Un segundo caso es, justamente, el de Ricardo Buryaile (1962), quien pertenece a una familia de productores agropecuarios de las provincias de Salta y Formosa con cierta trayectoria en el ruralismo de la región. Ricardo se recibió de contador en la Universidad Católica Argentina y volvió a su provincia para dedicarse a las actividades del campo. Allí llegó a ser presidente de la Sociedad Rural de Pilcomayo y titular de la Confederación de Sociedades Rurales de Chaco y Formosa, para más tarde en 2007 alcanzar la vicepresidencia segunda de CRA.

El conflicto de 2008 lo catapultó al conocimiento mediático y en 2009 encabezó la lista de diputados nacionales por Formosa del ACyS, ingresando a la Cámara por cuatro ańos. Al promediar su mandato en 2011, fue precandidato a gobernador de su provincia por la UCR, pero perdió en la interna. Reelegido diputado en 2013, compitió sin éxito en 2015 para la intendencia de la ciudad de Formosa, por Cambiemos. A finales de ese año, con el triunfo de Cambiemos a nivel nacional y la llegada de Mauricio Macri a la presidencia fue designado ministro de agroindustria, cargo que conserva hasta 2017. En 2019 se candidatea nuevamente para diputado nacional. 
En tercer término, encontramos al hijo del mayor productor de soja de la Argentina, Alfredo Olmedo (1965), quien es él también productor agropecuario en la provincia de Salta. Tuvo diversas ocupaciones en los emprendimientos de su padre hasta tener sus propios campos y empresas. Además, se dedicó durante varios años a la práctica de deportes como el automovilismo, el motocross y las carreras de lancha.

Su ingreso a la política se dio antes del conflicto con el campo cuando en 2007 ganó una banca en el Senado provincial. Su identificación con el sector, a pesar de no formar parte de ninguna de las entidades ruralistas, hizo que tanto la prensa como los trabajos académicos sobre el tema lo identificaran como agrodiputado, en tanto ganó su banca en las elecciones de 2009 cuando se presentó con su propio partido Salta Somos Todos (SST) y, a pesar de haber salido tercero, ingresó a la Cámara. Dos años después fue candidato a gobernador y perdió. Al finalizar su mandato en 2013 intentó ser senador nacional por Salta, quedando también afuera. En 2015 se postuló como vicegobernador del histórico dirigente del justicialismo salteño Juan Carlos Romero, aunque la fórmula fue finalmente derrotada. Meses después triunfaba nuevamente como diputado nacional, asumiendo con mandato hasta 2019, esta vez aliado al Frente Renovador ${ }^{10}$. En 2019 es precandidato a gobernador de Salta.

Cercano a estos perfiles también podríamos ubicar al entrerriano Alfredo De Ángeli (1957), productor agropecuario del sur de la provincia y dirigente de la FAA de Gualeguaychú. Proviene de una familia con fuerte militancia en el ruralismo y en el cooperativismo agrícola de la zona. Participó en las protestas en los años noventa, pero no fue hasta el conflicto con el campo en 2008 que cobró una gran notoriedad mediática gracias a su participación en diversos cortes de ruta, asambleas y manifestaciones.

A pesar de los ofrecimientos de distintos sectores del radicalismo entrerriano para ser candidato en 2009 y 2011, De Ángeli permaneció

I0 Espacio de orientación peronista conformado para las elecciones de 2013 en torno a la figura de Sergio Massa, antiguo jefe de gabinete de Cristina Fernández de Kirchner, y de un conjunto de intendentes de la provincia de Buenos Aires. Distanciado del kirchnerismo, construyó un sistema de alianzas heterogéneas en el interior del país. Se disuelve para las elecciones de 2019, en las que Massa y varios referentes de su espacio vuelven con Cristina Kirchner. 
en la FAA. En 2013 decidió competir tras percibir que su carrera política en la federación estaba cerca de su techo. Ese año asumió como senador nacional por Entre Ríos con mandato hasta 2019 tras salir segundo en las elecciones, encabezando una lista apoyada por el PRO y por sectores del peronismo no kirchnerista. Dos años más tarde se presentaba como candidato a gobernador por Cambiemos, pero resultaba derrotado.

Aquí la estructura de recursos con la que ingresaron a la actividad partidaria se componía principalmente de capitales extrapolíticos construidos tanto colectiva como individualmente. Por un lado, poseían un reconocimiento como miembros de las asociaciones del gremialismo rural a las que representaban y en nombre de las cuales habían intervenido en el conflicto. Por el otro, nuevamente, tenían un capital mediático fruto de su participación en las protestas y cortes de ruta.

El caso de Olmedo requiere hacer la salvedad que supone el hecho de que este candidato hizo su debut electoral con un partido nuevo creado y desarrollado en torno a su propia figura, aun cuando en elecciones posteriores también se presentó como parte de frentes más amplios que incorporaban distintos sectores del peronismo salteño. Otra excepción de este caso es la no pertenencia a ninguna entidad gremial ruralista. Sin embargo, al igual que los anteriores se beneficia de la doble representación simbólica que supone el ser visto como perteneciente al campo y como empresario exitoso.

Estos tres casos - o cuatro, si incluimos a De Ángeli - es el de representantes gremiales del campo que eran amateurs en el terreno de la política partidaria, pero activaron un proyecto personal de ingreso a la misma, plasmado en la continuidad que muestran en el ejercicio de cargos públicos electivos o no electivos una vez finalizado su mandato original en 2013. Incluso antes de que este terminara ya habían comenzado a candidatearse para otros puestos más codiciados como el de intendente o el de gobernador. Para ellos el conflicto agrario significó también una oportunidad de ingresar al campo político y el inicio de una carrera propiamente político partidaria.

No todos los casos fueron exitosos, sin embargo. El de Hilma Ré (1956) muestra un desarrollo similar en la búsqueda de continuar como diputada, 
aunque se trunca por resultados electorales adversos. Es ingeniera agrónoma de la Universidad Nacional de Entre Ríos y productora agropecuaria en la zona de Diamante, Entre Ríos. Su primer acercamiento a la política se había dado militando en el justicialismo de la provincia, del que se alejó a fines de los años 80. Entre 2005 y 2009 había sido presidenta de la Sociedad Rural de Diamante.

Para las elecciones de 2009 fue convocada por Patricia Bullrich de la CC-ARI para sumarse a la lista de diputados del ACyS. Se presentó como segunda candidata detrás de Benedetti e ingresó a la Cámara con mandato hasta 2013. Ese año buscó su reelección, pero la lista de UNEN obtuvo el tercer puesto y no ingresó ningún legislador. Continuó vinculada a la dirigente de Elisa Carrió y fue promovida por esta para ser precandidata a diputada en 2017, aunque finalmente quedó afuera en el armado de las listas. En 2019 se candidateó para diputada provincial, pero no llegó a ingresar a la Cámara.

En definitiva, se trata de actores para los que los sucesos de 2008 abrieron una oportunidad de comenzar sus carreras políticas "por arriba" a partir de su propulsión a las cabezas de lista para diputados nacionales, habiendo tenido poco o ningún involucramiento previo en la vida partidaria de las agrupaciones por las que se presentaron. Para ello hicieron valer sus credenciales extrapolíticas tanto colectivas - el activismo en la representación rural - como individuales - su conocimiento mediático.

\section{Estrellas fugaces en el Congreso: los gremialistas}

Un tercer conjunto de agrodiputados está conformado por aquellos que sólo ocuparon su banca en la Cámara Baja por un único mandato entre 2009 y 2013. Es decir, que no se candidatearon para ser reelegidos ni para ocupar nuevos puestos, sino que retornaron al gremialismo rural como principal actividad de representación ni bien finalizó su cargo en el Congreso. Consideramos el no haberse postulado en elecciones subsiguientes como indicador de que su apuesta principal se encontraba en la carrera dentro de sus entidades gremiales.

Aquí tenemos a Jorge Chemes (1956), productor lechero entrerriano que presidió la Sociedad Rural de Nogoya, integró la Cámara de 
Productores Lecheros de Entre Ríos, fue miembro de AACREA y fundó y presidió la filial Nogoyá de esa entidad. Para la época del conflicto con el campo presidía la Federación de Asociaciones Rurales de Entre Ríos y era miembro de CRA. En las legislativas de 2009, Chemes fue el tercero que ingresó como diputado en la lista del ACyS de Entre Ríos que llevaba también como candidatos a Benedetti y a Ré. Al finalizar su mandato en 2013 volvió a ser elegido presidente de FARER y dos años después vicepresidente primero de CRA.

Por su parte, Lucio Aspiazú (1959) proviene de una familia de productores ganaderos del sur de Corrientes. Fue presidente de la Sociedad Rural de Curuzú Cuatiá y de la Fundación Correntina de Sanidad Animal y consejero de CRA. En las elecciones de 2009 encabezó la lista del ACyS de Corrientes. Al finalizar su mandato en 2013 volvió de lleno a la producción agropecuaria y al gremialismo rural. En la actualidad es presidente de la Asociación Argentina de Criadores de Corriedale.

También encontramos a Pablo Orsolini (1955), productor agropecuario de Villa Ángela, Chaco, con militancia en el Partido Socialista (PS) y nutrida trayectoria en el gremialismo. Allí fue presidente de la filial local de la FAA entre 1982 y 1999 y representante de esa entidad en el Instituto Nacional de Tecnología Agropecuaria (INTA). También se desempeñó como presidente de la Cooperativa Agrícola Regional Villa Ángela y vicepresidente de la federación de cooperativas FeCoFe. A partir de 2000 asumió como vicepresidente primero de la FAA y en 2002 fue nombrado director del Registro Nacional de Trabajadores Rurales y Empleadores (RENATRE) en representación de su entidad y luego su presidente a partir de 2005. Para las legislativas de 2009 encabezó la lista del ACyS-Frente de Todos en el Chaco e ingresó como diputado nacional con mandato hasta 2013. Al ańo siguiente intentó ser presidente de la FAA, quedando segundo y como principal referente de la oposición interna.

Por su parte, como militante del radicalismo pampeano, Ulises Forte (1962) ocupó diversos cargos en la UCR provincial desde la década del 80. Su participación en la FAA en tanto productor agropecuario de la zona data de la misma época y lo llevaría por distintos cargos hasta ser vicepresidente segundo a nivel nacional entre 2005 y 2009. El conflicto 
con el campo lo encontró en un rol muy activo en las manifestaciones y cortes de ruta. En 2009 fue precandidato a senador nacional, pero perdió la interna con Juan Carlos Marino y se postuló para diputado encabezando la lista del ACyS. Triunfó y ocupó su banca hasta 2013, cuando volvió de lleno a la actividad gremial. Fue designado representante de la FAA en el Instituto de Promoción de la Carne Vacuna Argentina y, a partir de 2015, su presidente.

Finalmente, presidenta de la FA de Río Cuarto, Córdoba, e integrante del Movimiento de Mujeres Agropecuarias en Lucha, Estela Garnero (1958) es una productora agropecuaria del sur cordobés que adquirió notoriedad con los cortes de rutas durante el conflicto con el campo en 2008. Como consecuencia, fue convocada por el oficialismo de la provincia a cargo de Juan Schiaretti - que venía de romper con el gobierno kirchnerista para formar un espacio peronista disidente - a los fines de ocupar el segundo lugar en la lista de candidatos a diputados de Unión por Córdoba (UC), a pesar de no haber tenido ninguna militancia previa en ese espacio político. Ingresó a la Cámara con mandato hasta 2013 y no volvió a candidatearse desde entonces.

Estos agrodiputados poseen una estructura de capitales similar a la de los amateurs con proyecto político - reconocimiento como parte del ruralismo y conocimiento mediático -, pero, aun si en algunos casos han tenido una militancia partidaria, los diferencia el hecho de que para ellos la principal apuesta se encontró en las organizaciones rurales y no en los partidos. El conflicto de 2008 significó su ingreso a la política, pero no el inicio de una carrera en la arena partidaria.

\section{Procedencias múltiples, destinos divergentes}




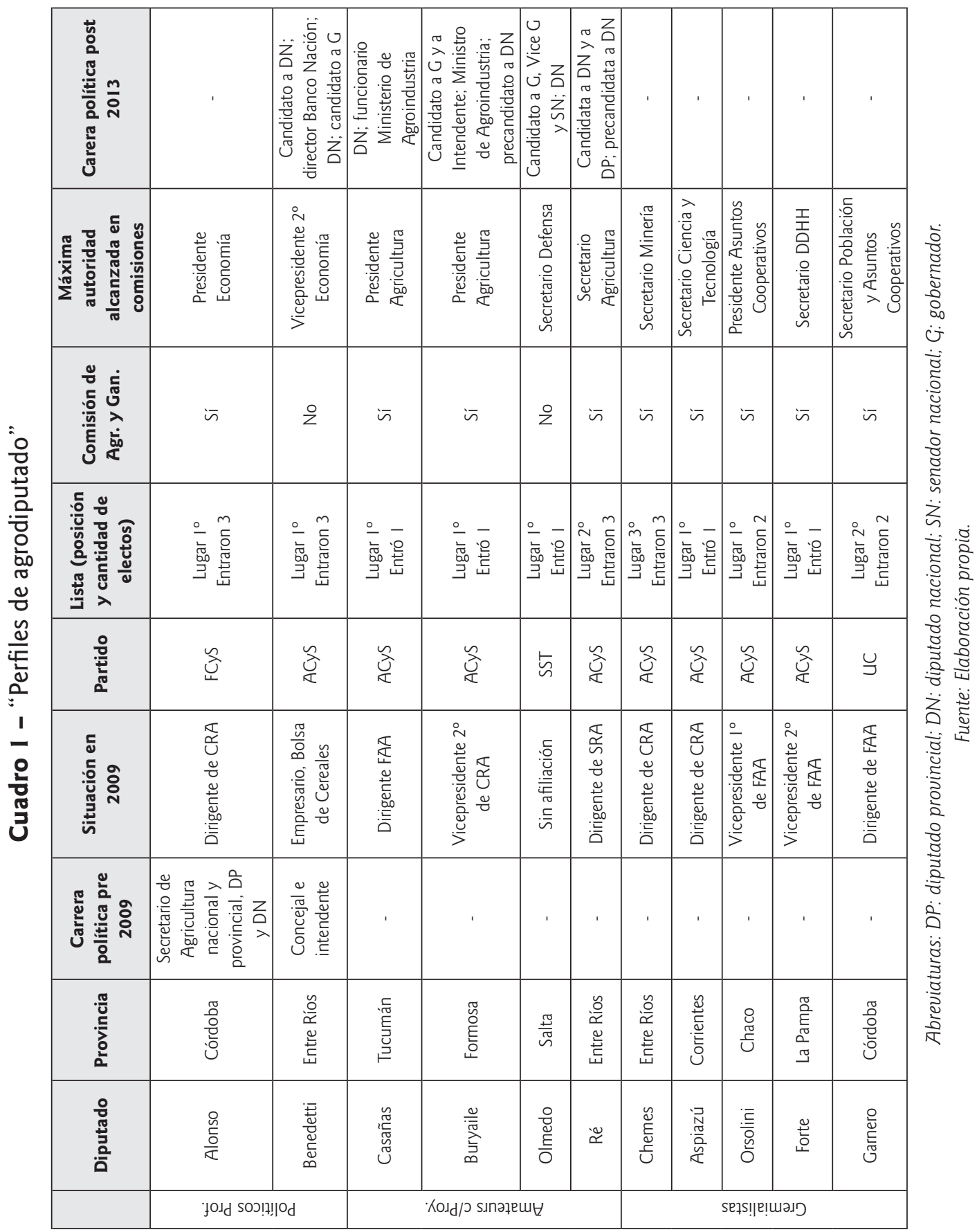


El cuadro 1 sintetiza una serie de variables que da cuenta de las diferencias entre los tres perfiles de agrodiputados. En primer lugar, los políticos profesionales tienen una carrera política preexistente con varios cargos previos y, en uno de los casos, también posteriores. Es decir que la diputación 2009-2013 se encastró en una sucesión más o menos interrumpida de distintos puestos. En cuanto a las comisiones, Agricultura y Ganadería no pareció ser especialmente codiciada, en tanto sólo uno formó parte de esta y a título de vocal, mientras que ambos fueron autoridades en la comisión de Economía. Por su parte, los amateurs con proyecto no poseen cargos políticos antes de 2009, pero sí han ocupado o buscado ocupar diversos puestos legislativos y ejecutivos, así como locales, provinciales y nacionales luego de finalizar su mandato o, incluso, durante el mismo. En otras palabras, su banca en 2009 fue el puntapié de una carrera política que se forjó al calor del conflicto agrario. Además, sobre cuatro diputados, tres ocuparon puestos de autoridad en la comisión de Agricultura. Finalmente, los gremialistas no ocuparon cargos políticos antes de 2009 ni después de 2013. Su carrera político partidaria se limitó a la diputación que analizamos. Muestran además una posición más marginal en el Congreso en comparación con los grupos anteriores, como lo demuestra el que tengan una menor proporción de cabezas de lista y que no hayan sido autoridad ni en la comisión de Agricultura ni en ninguna de las consideradas centrales (Presupuesto y Hacienda, Asuntos Constitucionales, Relaciones Exteriores, Economía, entre otras)"

Cuadro 2 - "Recursos movilizados por cada perfil de agrodiputado"

\begin{tabular}{|c|c|c|c|c|}
\hline & \multicolumn{2}{|c|}{ Político } & \multicolumn{2}{|c|}{ Extra-político } \\
\hline & Colectivo & Individual & Colectivo & Individual \\
\hline \begin{tabular}{|l|} 
Políticos \\
profesionales
\end{tabular} & $\begin{array}{l}\text { Son "hombres de } \\
\text { partido" }\end{array}$ & \multirow{3}{*}{$\begin{array}{l}\text { Poco o nulo (a } \\
\text { excepción de } \\
\text { Olmedo) }\end{array}$} & \multirow{3}{*}{$\begin{array}{l}\text { Miembros de } \\
\text { organizaciones } \\
\text { ruralistas o entidades } \\
\text { patronales del sector }\end{array}$} & \multirow{3}{*}{ Mediático } \\
\hline $\begin{array}{l}\text { Amateurs con } \\
\text { proyecto }\end{array}$ & $\begin{array}{l}\text { Poca o ninguna } \\
\text { cercanía previa con }\end{array}$ & & & \\
\hline Gremialistas & la política partidaria & & & \\
\hline
\end{tabular}

Fuente: Elaboración propia.

I L La única excepción es la de Orsolini, quien presidió la comisión de Asuntos Cooperativos, Mutuales y de ONG. Esta constituye una comisión de segundo orden, pero es significativa para él, en tanto se encuentra vinculado al cooperativismo en su provincia. 
El análisis al nivel de los recursos que cada uno movilizó en ocasión de presentarse a las legislativas de 2009 se encuentra sintetizado en el cuadro 2 y muestra nuevas diferencias entre los tres perfiles. Primeramente, los políticos profesionales son, ante todo, representantes de una empresa política colectiva (GAXIE, 2004), "hombres de partido" que cuentan con un capital en su pertenencia partidaria, traducido en saberes, redes, prácticas y estructuras disponibles para competir en elecciones. Pero el conflicto con el campo también los dota de un reconocimiento como parte de ese colectivo, que comparten todos los agrocandidatos. La principal diferencia con los demás se encuentra en que, tanto los amateurs con proyecto como los gremialistas, carecen en líneas generales de ese capital del militante partidario, aunque todos se postularon por partidos ya existentes o por alianzas que agrupan a distintos partidos ya implantados y con sus propias estructuras. De todos modos, formar parte de distintas organizaciones ruralistas también constituye un recurso colectivo que supone toda una serie de saberes acerca de las actividades de representación, prácticas de negociación, competencia en elecciones y otras que, si bien difieren de las estrictamente partidarias, se le asemejan.

Ahora bien, en líneas generales, aceptar una candidatura puede ser visto como una apuesta en la que se consiente en invertir una determinada cantidad de recursos, tiempo y esfuerzo. Si se gana el cargo para el cual se postuló, las actividades que se ejercían antes de asumir se verán en algún modo postergadas. De allí que toda inversión en la competencia partidaria pueda ser vista al mismo tiempo como una desinversión en el espacio del cual proviene el actor.

Para el caso de los amateurs con proyecto y los gremialistas, el peligro de aceptar una postulación no residió tanto en el posible descuido de sus actividades productivas como en la probable pérdida de espacios en las organizaciones ruralistas, para el caso de los que en 2009 se encontraban más activos en ellas. En tanto empresarios agropecuarios, aun cuando descuiden su actividad, siguen recibiendo ganancias de sus campos antes, durante y después de su paso por la política partidaria. Pero como dirigentes ruralistas, el ingreso a la política puede comportar mayor riesgo, a menos que se haya encontrado un "techo" al crecimiento dentro de la organización o se perciba el involucramiento partidario como un recurso a hacer valer con posterioridad. Estas decisiones suponen implementar 
estrategias que privilegien la carrera político partidaria, la carrera dentro de la organización patronal o intentar conciliar ambas (OFFERLÉ, 2013).

Como toda tipología supone "estilizar" o "acentuar" algunos delos rasgos constatados en la realidad en beneficio de la posibilidad de categorizar y elaborar hipótesis de trabajo, corresponde remarcar ciertas particularidades. El caso de Alfredo Olmedo es particular en tanto no pertenece a ninguna organización ruralista y posee un tipo de capital político individual en la medida que se asemeja al empresario político individual (GAXIE, 2004), dueño de sus propios medios y de un reconocimiento ligado a su persona antes que a un partido u organización. En segundo lugar, Alonso continúa formando parte de CRA, aunque su principal activismo en dicha entidad haya sido en las décadas del ochenta y el noventa. Esto también supone un capital extrapolítico de tipo colectivo, no obstante para 2009 se encuentra mucho más ligado a la política partidaria que a la representación gremial. Finalmente, tanto Forte como Orsolini y Ré fueron militantes o estuvieron ligados a las estructuras partidarias del radicalismo, el socialismo y el peronismo primero y la CC después, respectivamente.

\section{Reflexiones finales}

La cuestión de la participación de los dirigentes agropecuarios en las listas de candidatos al Congreso en 2009 había sido abordada principalmente desde la ciencia política a partir de la pregunta por la coordinación electoral entre las entidades del agro y los partidos políticos, mostrando cómo no habían sido las elites ruralistas las que habían buscado apoyo a candidaturas propias en los partidos, sino que fueron estos últimos los que se lanzaron a buscar candidatos del agro para cosechar un mayor caudal de votos (DEL COGLIANO, 2016). Analizando las trayectorias y carreras de cada uno de estos actores ampliamos el conocimiento sobre el tema revelando las heterogeneidades de este conjunto de actores y distinguiendo tres perfiles de agrodiputados en función de sus trayectorias y de los recursos que movilizaron para llegar al Congreso en 2009.

En este sentido, las fronteras del espacio político argentino muestran cierta porosidad al permitir el ingreso de agentes en principio ajenos a las lógicas partidarias, aunque, como vimos, diestros en la práctica de la 
representación de intereses. El creciente desprestigio del parlamento y de la actividad política en general vuelve más plausible el ingreso de estos actores, en tanto los partidos buscan legitimarse frente al descrédito imperante, no obstante son los momentos de mayor turbulencia política - entre los cuales el conflicto con el campo en 2008 es quizás uno de los mayores ejemplos en la Argentina de los últimos años - cuando la oportunidad de ingresar a la competencia electoral aumenta para aquellos que se presentan por primera vez (CANON, 1990).

Ahora bien, dicha apertura, que incluye la incorporación de estos actores, no mengua el peso de las dinámicas estrictamente políticas. Recordémoslo. La primera mitad de los mandatos de estos diputados, entre 2009 y 2011, transcurrió en un Congreso en el que ni el oficialismo ni la suma de las fuerzas de la oposición contaban con mayoría propia. Esto despertó en los partidos opositores ciertas esperanzas de doblegar al gobierno, que pronto se vieron naufragadas ante un parlamento trabado y con poca iniciativa. Luego, tras las elecciones de 2011 en las que Cristina Kirchner resultó reelegida por una amplia diferencia, el kirchnerismo recuperó sus mayorías en ambas Cámaras y la segunda mitad de los mandatos de estos diputados transcurrió en posiciones de clara minoría. Ambos momentos representaron escenarios muy poco favorables para la promoción de los reclamos sectoriales que pretendían impulsar.

Para estos candidatos, el conflicto con el campo significó una oportunidad, ya sea de volver a competir y triunfar en elecciones como de hacerlo por primera vez. Pero los capitales extrapolíticos, el llegar "desde afuera" o el ser identificado con determinado sector por fuera de la política, sólo constituyó un recurso al comienzo. Luego, la política impone sus propias lógicas y dinámicas, con lo que las condiciones de continuidad, interrupción o fin de sus carreras, así como el desempeńo en su cargo, ya no dependen del reconocimiento adquirido en protestas que quedaron lejos en el tiempo. Estas lógicas son bien conocidas por los políticos profesionales y rápidamente aprendidas tanto por los amateurs con proyecto como por los gremialistas, aunque de manera relativamente exitosa para los primeros y frustrante para los segundos.

En cada una de las elecciones legislativas posteriores volvieron 
a presentarse con suerte dispar otros candidatos provenientes de organizaciones ruralistas, evidenciando que la identificación con el sector agropecuario se convertiría después de 2008 en un recurso a hacer valer en el ingreso a la competencia electoral. No obstante, el escenario de 2009 presenta la particularidad de que dicho capital irrumpe de manera novedosa y tras un hecho de gran conmoción.

Mientras que las consecuencias del conflicto con el campo en la composición de las elites políticas argentinas parecen bastante limitadas desde el punto de vista cuantitativo, el estudio del ingreso a la política de dirigentes del mundo agropecuario permite comprender las relaciones entre las elites políticas y las elites económicas, tanto como las condiciones de reconversión de los recursos individuales y colectivos en capital político en la Argentina contemporánea.

\section{Referencias}

AGROSÍTIO. Los agrodiputados van divididos al Congreso. 30 nov. 2009. Disponible en: http:// www.agrositio.com/vertext/vertext.php?id=107548\&se=. Acceso en: 5 feb. 2018.

ARMELINO, M. Los sindicalistas en el parlamento argentino (2013-2015). Revista Agenda Política, São Carlos, v. 4, n. 3, p. 267-290, sep./dic. 2016.

BOLTANSKI, L. L'espace positionnel: multiplicité des positions institutionnelles et habitus. Revue Française de Sociologie, París, v. 14, n. 1, p. 3-26, 1973.

BRUÈRE, M-H.; GAXIE, D. Le recrutement extra-parlementaire des ministres sous la Ve République. DEROSIER, J.-P.; SACRISTE, G. (dir.). L'État, le Droit, le Politique Mélanges en l'honneur de Jean-Claude Colliard. París: Dalloz: 2014. p. 339-349.

CANELO, P. Acerca de la construcción de carreras políticas en la Argentina. Los senadores nacionales en 1973, 1983 y 1989. Polhis, v. 4, n. 7, p. 140-153, 2011.

CANELO, P. Ministros políticos y ministros técnicos. La cuestión de la Defensa en la Argentina desde 1966 a la actualidad. In: CANELO, P.; HEREDIA, M. (comp.). Los puentes y las puertas. Las fronteras de la política argentina a través de sus elites. San Martín: Universidad Nacional de San Martín, 2019. p. 157-180.

CANON, D. T. Actors, Athletes, and Astronauts. Political Amateurs in the United States Congress. Chicago: University of Chicago Press, 1990.

CLARÍN. Sólo quedarán 2 'agrodiputados' de los 11 que entraron en 2009. 29 oct. 2013. Disponible en: https://www.clarin.com/politica/solo-quedaran-agrodiputados-entraron_0_SJCl3nGoPmg. html. Acceso en: 6 feb. 2018. 
DAMIN, N. Del sindicato al parlamento. La profesionalización política de dirigentes sindicalespolíticos en la Argentina del siglo XX. Nuevo Mundo Mundos Nuevos, 2011 [en línea]. Disponible en: https://journals.openedition.org/nuevomundo/62081 Acceso en: 16 ago. 2017.

DANDAN, A. La cosecha de los agrodiputados. Página 12, 30 junio 2009. Disponible en: https:// www.pagina12.com.ar/diario/elpais/1-127501-2009-06-30.html. Acceso en: 5 feb. 2018

DE LUCA, M.; MALAMUD, A. Argentina: turbulencia económica, polarización social y realineamiento político. Revista de Ciencia Política, v. 30, n. 2, p. 173-189, 2010.

DEL COGLIANO, N. C. Después del conflicto con el campo en 2008, ¿̨los partidos abrieron sus listas a los dirigentes agropecuarios? Un estudio sobre la coordinación electoral de elites partidarias y agropecuarias en Argentina. Desarrollo Económico, v. 56, n. 218, p. 101-130, 2016.

DONATELLO, L. M. Legisladores Representantes Empresariales en Argentina y Brasil: la emergencia de una categoría de especialistas. Sociologia e Política, v. 25, n. 63, p. 139-158, 2017.

DONATELLO, L. M.; LEVITA, G. ¿¿Renovación de las elites o renovación de las élites políticas? Los diputados outsiders en los países del Mercosur (2003-2015). Revista de Investigaciones Políticas y Sociológicas, v. 16, n. 2, p. 45-64, 2017.

FERRARI, M. Algunas reflexiones acerca del proceso de profesionalización política. Aspectos generales y observaciones sobre la Argentina del siglo XX. In: PÉREZ, G.; AELO, O.; SALERNO, G. (ed.). Todo aquel fulgor. La política argentina después del neoliberalismo. Buenos Aires: Nueva Trilce, 2011.

GAXIE, D. Apréhensions du politique et mobilisations des expériences sociales. Revue Française de Science Politique, v. 52, n. 2-3, p. 145-178, 2002.

GAXIE, D. La democracia representativa. Santiago de Chile: LOM, 2004.

GENÉ, M. En torno a los profesionales de la política. Trayectorias, prácticas y destrezas en el ejercicio del poder político desde el estado. Revista Perspectivas de Políticas Públicas, v. 1, n. 1, p. 85-107, 2011.

GENÉ, M. Los ministerios políticos del gabinete nacional: perfiles y puertas de entrada de sus elites en democracia. In: CANELO, P.; HEREDIA, M. (comp.). Los puentes y las puertas. Las fronteras de la política argentina a través de sus elites. San Martín: Universidad Nacional de San Martín, 2019. p. 209-233.

GIORGI, G. Los factores “extrapolíticos” de la carrera política: Una aproximación a las sociabilidades de los ministros de la Nación en la Argentina (1854-2011). Política - Revista de Ciencia Política, v. 52, n. 2, p. 243-275, 2014 .

GRASS, C. Empresarios rurales y acción política en Argentina. Estudios Sociológicos, v. 30, n. 89, p. 459-487, 2012.

HEREDIA, M.; GENÉ, M.; PERELMITER, L. Hacia una socio-historia del Gabinete Nacional.

Polhis, v. 5, n. 9, p. 284-290, 2012. 
HORA, R. La crisis del campo del otoño de 2008. Desarrollo Económico, v. 50, n. 197, p. 81111, 2010.

JOIGNANT, A. Habitus, campo y capital. Elementos para una teoría general del capital político. Revista Mexicana de Sociología, v. 74, n. 4, p. 587-618, 2012.

LA NACIÓN. Los agrodiputados pelean espacios clave. 8 dic. 2009. Disponible en https://www. lanacion.com.ar/1209201-los-agrodiputados-pelean-espacios-clave. Acceso en: 5 feb. 2018.

LANDAU, M. Campo político y elencos legislativos en la Ciudad de Buenos Aires (1997-2011): un análisis sobre la renovación legislativa y política local. Congreso Internacional Élites y liderazgo en tiempos de cambio. Salamanca: [s. n.], 2015.

LASCURAIN, M. C. Mundo sindical, confianza y expertise: los puentes de acceso a la elite ejecutiva en Santa Fe (1983-2007). In: CANELO, P.; HEREDIA, M. (comp.). Los puentes y las puertas. Las fronteras de la política argentina a través de sus elites. San Martín: Universidad Nacional de San Martín, 2019. p. 129-153.

LATTUADA, M. Acción Colectiva y Corporaciones Agrarias en la Argentina. Transformaciones Institucionales a Fines del Siglo XX. Bernal: Editorial de la Universidad Nacional de Quilmes, 2006.

LEIRAS, M.; CRUZALEGUI, I. Argentina: problemas macroeconómicos, conflicto social y debilitamiento de la coalición de gobierno. Revista de Ciencia Política, v. 29, n. 2, p. 223-246, 2009.

LEVITA, G. La política como profesión: perfiles y tipos de trayectorias de los senadores argentinos. Telos, v. 17, n. 1, p. 38-57, 2015.

LEVITA, G. ¿De patricios a plebeyos? Sociología de las elites políticas. El Senado y los senadores argentinos en la primera década del siglo XXI. Estudios Políticos, Medellín, n. 51, p. 13-35, 2017.

LEVITA, G. Llegar a la Cámara de Diputados de Argentina “desde afuera”: recursos, inversiones y marginalidades. Rev. Sociol. Polit., v. 27, n. 71, p. e001, 2019.

MELLADO, V. La representación política en cuestión. Trayectorias de los legisladores de Mendoza en el largo plazo (1946-1999). In: MAURO, S.; ORTIZ DE ROZAS, V.; PARATZ, M. (comp.). Política Subnacional en Argentina. Enfoques y problemas. Buenos Aires: CEAP-Honorable Senado de la Nación, 2016. p. 143-177.

MUSTAPIC, A. M. Los partidos políticos en la Argentina: condiciones y oportunidades de su fragmentación. In: ACUÑA, C. H. (comp.). ¿Cuánto importan las instituciones? Gobierno, Estado y actores en la política argentina. Buenos Aires: Siglo XXI, 2013. p. 249-290.

NARDACCHIONE, G.; TARABORELLI, D. La importancia de los aliados: un estudio sobre el conflicto rural (marzo-julio 2008). In: ARONSKIND, R.; VOMMARO, G. (Comp.). Campos de batalla. Las rutas, los medios y las plazas en el nuevo conflicto agrario. Buenos Aires: Prometeo, 2010. p. 119-152.

OFFERLÉ, M. Entrées en politique. Politix, v. 9, n. 35, p. 3-5, 1996. 
OFFERLÉ, M. Professions et profession politique. In: OFFERLÉ, M. (dir.). La profession politique XIXe-XXe siècles. París: Belin, 1999. p. 7-35.

OFFERLÉ, M. Los partidos políticos. Santiago de Chile: LOM, 2004.

OFFERLÉ, M. Los oficios, la profesión y la vocación de la política. PolHis, v. 1, n. 7, p. 84-98, 2011.

OFFERLÉ, M. Les patrons des patrons. Histoire du Medef. París: Odile Jacob, 2013.

ORTIZ DE ROZAS, V. Las formas de reclutamiento del personal político, una vía de entrada al estudio del régimen político provincial. Santiago del Estero (1999-2009). Revista Perspectivas de Políticas Públicas, v. 1, n. 1, p. 133-159, 2011.

PERELMITER, L. La constitución de una autoridad plebeya. El ministerio "de la pobreza" en la Argentina reciente. Polhis, v. 5, n. 9, p. 309-318, 2012.

RODRIGO, C. Actores, relaciones y escaños. Apuntes para el estudio de las carreras políticas de los diputados nacionales por la Provincia de Buenos Aires. Estudios, n. 32, p. 135-148, 2014.

SOSA, P. Los dirigentes del Frente para la Victoria Santacruceña: una elite de militantes (19882003). In: CANELO, P.; HEREDIA, M. (comp.). Los puentes y las puertas. Las fronteras de la política argentina a través de sus elites. San Martín: Universidad Nacional de San Martín, 2019. p. 109-128.

TISSOT, S. Les reconversions militantes. In: TISSOT, S., GAUBERT, C.; LECHIEN, M-H. (ed.). Reconversions militantes. París: Pulim, 2004. p. 9-17.

TORRE, J. C. Los huérfanos de la política de partidos. Sobre los alcances y la naturaleza de la crisis de representación partidaria. Desarrollo Económico, v. 42, n. 168, p. 647-665, 2003.

TULA, M. I.; DE LUCA, M. Reglas electorales y dinámicas políticas en la selección de candidatos. Cambios y continuidades de Alfonsín a los Kirchner. In: MALAMUD, A.; DE LUCA, M. (coord.).

La política en tiempos de los Kirchner. Buenos Aires: Eudeba, 2011. p. 73-84.

VOMMARO, G. La larga marcha de Cambiemos. La construcción silenciosa de un proyecto de poder. Buenos Aires: Siglo XXI, 2017.

VOMMARO, G.; ARMESTO, M. ¿Nuevos políticos en el partido, viejos políticos en las listas? Reclutamiento partidario y división del trabajo político en PRO, en la Ciudad Autónoma de Buenos Aires. Pasado Abierto, n. 2, p. 110-132, 2015.

WEBER, M. La política como profesión. In: WEBER, M. La ciencia como profesión - La política como profesión. Madrid: Austral, 2007. p. 82-102. 


\section{Professionals, amateurs and rural associations' leaders. Trajectories and entries to politics of the "agro-deputies" in the Argentine Congress}

\section{Abstract}

This article is framed into a political sociology of elites and looks into political entries in nowadays Argentina through the social, unionist and political trajectories of the Argentine national congressmen elected in 2009 who came from the agricultural entrepreneurs unions, known as "agrodiputados". It achieves it from a qualitative perspective and using in-depth interviews and secondary sources. We show how these congressmen, who had been studied since their common features, are in fact a heterogeneous group where we can find three different profiles. We analyse their entrances into politics and the conversion of their resources into political capital. We show how coming "from outside" only counted as a resource at the beginning of their career. Then, professional politics logic prevailed.

Keywords: Congressmen. Entering politics. Political capital. Political careers. Argentina. 